\title{
Robust Laser Beam Tracking Control using Micro/Nano Dual-Stage Manipulators
}

\author{
Nabil Amari, David Folio and Antoine Ferreira
}

\begin{abstract}
This paper presents a study of the control problem of a laser beam illuminating and focusing a microobject subjected to dynamic disturbances using light intensity for feedback only. The main idea is to guide and track the beam with a hybrid micro/nanomanipulator which is driven by a control signal generated by processing the beam intensity sensed by a four-quadrant photodiode. Since the pointing location of the beam depends on real-time control issues related to temperature variation, vibrations, output intensity control, and collimation of the light output, the 2-D beam location to the photodiode sensor measurement output is estimated in real-time. We use the Kalman filter (KF) algorithm for estimating the state of the linear system necessary for implementing the proposed track-following control approach. To do so a robust master/slave control strategy for dual-stage micro/nanomanipulator is presented based on sensitivity function decoupling design methodology. The decoupled feedback controller is synthesized and implemented in a 6 dof micro/nanomanipulator capable of nanometer resolution through several hundreds micrometer range. A case study relevant to tracking a laser-beam for imaging purposes is presented.
\end{abstract}

\section{INTRODUCTION}

High-precision position measurement systems based on laser beam reflection and/or transmission are commonly used in nanorobotics applications. It is composed of the optical detecting set, including the laser diode (LD), the positionsensitive detector (PSD), alignment mechanisms, and the frame structure for maintaining the optical configuration. The general problem is to focus the beam in few micrometer size spots and to control actively the beam direction to stabilize the beam at a desired location. It is desirable in nanomanipulation tasks (see Figure 1) when focusing a nearinfrared laser beam at a nerve cells leading edge [1], [2], when the laser beam perfectly tracks the moving atomic force microscope (AFM) probes [3] during manipulation tasks, or when the laser beam illuminates a microobject handled by a nanogripper for material characterization [4]. Usually, the laser beam calibration is time-consuming since the laser beam could be steered manually. Precise laser beam tracking of dynamic position with high-bandwidth rejection of disturbances produced by nanomanipulator platform vibration, piezoelectric actuator thermal drifts, photodetector noises, brownian motion of laser beam and atmospheric turbulence are critical for the success of micro and nanomanipulation tasks. As the single photodiode sensor is currently being used only for position measurement, the possibility of using it

N. Amari, D. Folio and A. Ferreira are with the Laboratoire PRISME EA 4229; Ecole Nationale Suprieure d'Ingnieurs de Bourges, 88 boulevard Lahitolle, 18020 Bourges, France. Corresponding author: Antoine Ferreira (Email:antoine.ferreira@ensi-bourges.fr, Tel: +33 24848 4079)

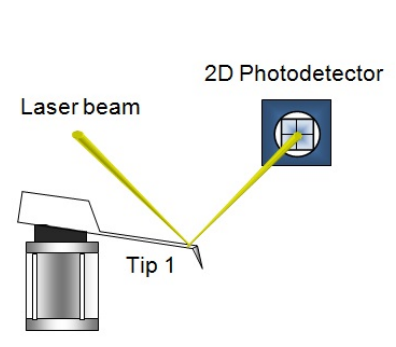

(a)

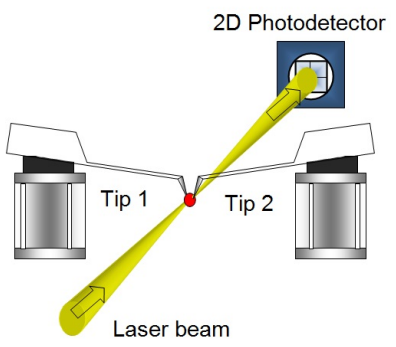

(b)
Fig. 1. High-precision position measurement based on laser beam (a) reflection and (b) transmission are commonly used in nanomanipulation applications.

for feedback control is of great interest, since this might significantly increase the overall performance and reliability of nanorobotic systems. Recently, two laser beam tracking configurations are found based in the literature, i.e. steering the laser beam or the photodetector. In the first case, some works propose to use fast tilt two-axis steering mirrors based on electrostatic MEMS actuators [5] or piezoelectric actuators with a fixed four-quadrant PSD. In the second case, the PSD is driven by a dual actuation system with robot micro/nanomanipulators [6], or $x-y$ linear positioning stages [7]. Whatever the technology involved, robust control of the laser beam tracking system is needed. The purpose of this paper is to design a control system that rejects disturbances in the sense of minimizing the variance of the error in the position of the laser beam. The main idea is to track the emitting beam by processing the maximum beam intensity sensed by a four-quadrant PSD mounted on a 6 dof dualstage micro/nanomanipulator platform. Since the pointing location of the beam depends on real-time control issues related to disturbances, the laser beam position is estimated in real-time using the Kalman filter (KF) algorithm. To do so, a robust decoupled design controller is presented based on sensitivity function decoupling design methodology. The decoupled feedback controller is synthesized and implemented in a 6 dof coupled magnetic and piezoelectric manipulation platform.

The paper is divided into five sections. Section 2 describes the experimental setup. Section 3 describes the dynamics modeling and system identification procedure and results. Section 4 describes the decoupled control design structure. Section 5 presents experimental results for the performance of the beam steering system. 


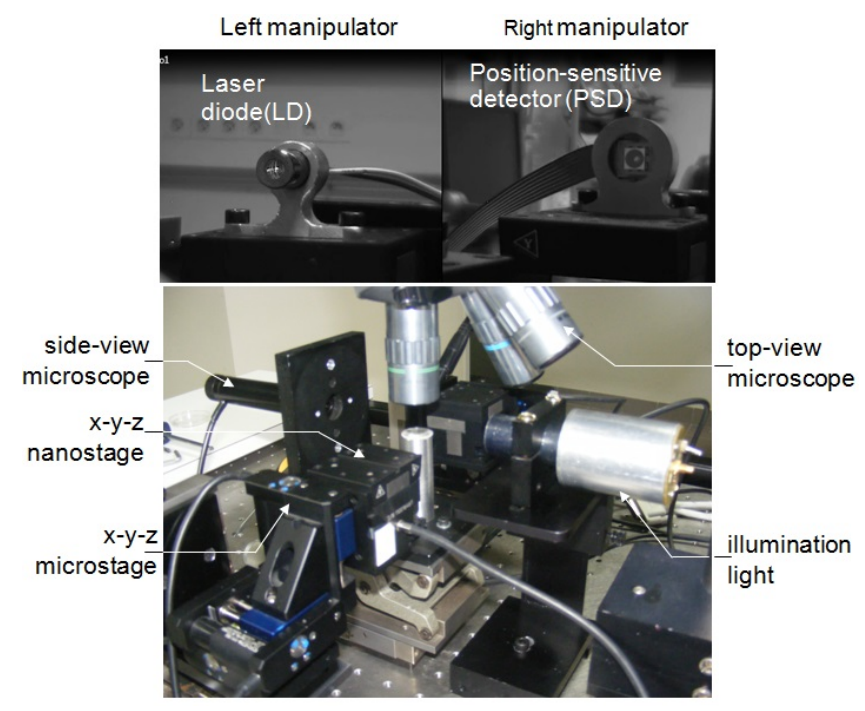

Fig. 2. Experimental setup.

\section{EXPERIMENTAL SETUP}

The control scheme of the beam pointing and tracking is shown in Fig.2. Two controllable micro/nano manipulators facing each other, composed of 3 d.o.f high-precision dualstages, i.e., magnetic X-Y-Z closed-loop microstage (MCL Nano-Bio2M on the $x-y-z$ axes) and piezoelectric $x-y-z$ closed-loop nanostage (P-611.3S NanoCube from Physics Instruments), respectively. The coarse motion of the microstage is about few centimeters and the fine motion of the nanostage is about $100 \times 100 \times 100 \mu \mathrm{m}$ positioning and scanning range comes in an extremely compact package. The laser source is mounted on top of the nanostage (right manipulator) producing the laser beam. The main components of the beam steering experiment are a $635 \mathrm{~nm}$ laser. A fourquadrant position sensing device (PSD) mounted on top of the nanostage (left manipulator) that measures the position of the image that the laser beam forms on a fixed plane. On the side view, a white light illuminates the workspace for top-view (top optical microscope - Mituyo $\times 50$ ) and sideview (TIMM $\times 150)$ imaging. The sample platform is at rest during manipulation that is fixed on the system base.

Fig. 3 shows the overall control scheme for power, laser beam tracking and micro/nano manipulator control. The laser beam motion control (Brownian or stochastic trajectory) and measurement sequences are processed in real-time using MATLABs XPC software with a stand alone target machine operating at a sample-and hold rate of $2 \mathrm{kHz}$. A data acquisition (DAQ) (NI 6289) card is used for highspeed capturing of photodiode voltage output from a lock-in to detect laser beam intensity maximum and beam tracking. A multi-thread planning and control system is developed to independently manage the coordination during parallel laser beam motion and tracking, respectively.

\section{DYNAMICS MODELING}

This section reviews the different model dynamics of the different system components.

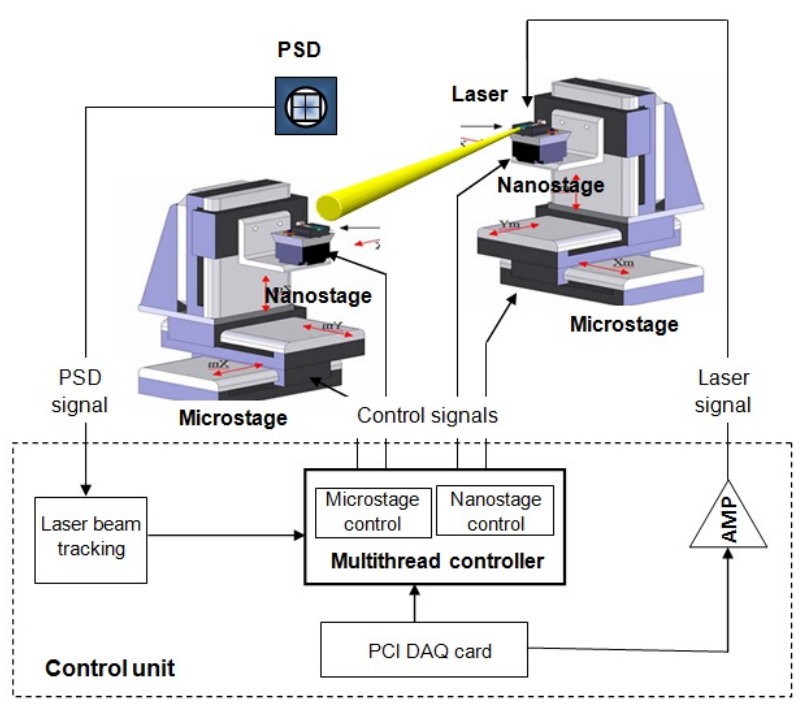

Fig. 3. Schematic diagram of the architecture of the laser beam tracking control system.

\section{A. Dynamics of Piezoelectric and Magnetic Actuators}

The first step for controller synthesis is to set up dynamic modeling. Because we have no parametric information on drivers of the dual micro/nano stages composed by piezoelectric 3-dof nanostage and the magnetic 3-dof microstage (that are deemed as three-input and two-output system), the modeling approach is based on system identification by experiment responses of micro/nano stages using Pseudo Random Binary Sequence input signal. The dynamics models of the micro and nano are formulated respectively by the following estimated transfer functions:

$$
\begin{aligned}
& G_{\text {micro }(x, y, z)}(z)=\frac{b_{0}+b_{1} z^{-1}+b_{2} z^{-2}+b_{3} z^{-3}}{1+a_{1} z^{-1}+a_{2} z^{-2}+a_{3} z^{-3}} \\
& G_{\text {nano }(x, y, z)}(z)=\frac{b_{1} z^{-1}+b_{2} z^{-2}+b_{3} z^{-3}}{1+a_{1} z^{-1}+a_{2} z^{-2}+a_{3} z^{-3}}
\end{aligned}
$$

The tracking processus of the laser beam motion detected by a four quadrant photo sensitive detector need a cooperation of micro stage and nano stage to ensure the tracking with high precision, where the tracking output signal generated by dual micro/nano stages is considered the sum of the positions outputs of the microstage and the nanostage due the strategy of control, more details are given in section 4.

\section{B. Dynamics of Four Quadrant Detector}

A four quadrant photo sensitive detector (PSD) has four photosensing parts arranged in four quadrants, respectively. When the elements are lighted by a beam of laser, they will generate currents according to the light intensity and then amplified into voltage signals. The combinations of voltages $V_{1}$ to $V_{4}$ can be used to indicate the offsets of the spot in relation to the center of the PSD as follows: 

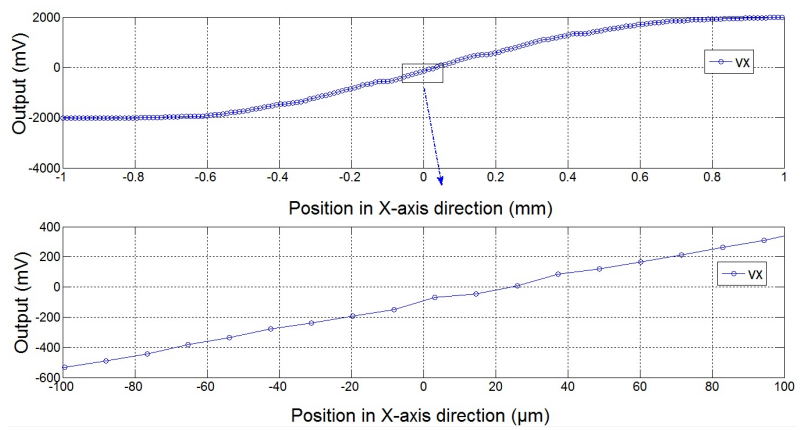

Fig. 4. Output voltage curve $V_{x}$ with a zoom in the block area near zero on an four-quadrant PSD.

$$
\begin{aligned}
& V_{x}=\left(V_{1}+V_{4}\right)-\left(V_{2}+V_{3}\right) \\
& V_{y}=\left(V_{1}+V_{2}\right)-\left(V_{3}+V_{4}\right) \\
& V_{s}=V_{1}+V_{2}+V_{3}+V_{4} .
\end{aligned}
$$

The $V_{x}$ and $V_{y}$ channel outputs are directly related to the energy of the laser beam that falls in each quadrant while $V_{s}$ is the sum voltage. It is assume that the light intensity on the laser's beam cross section obeys Gaussian distribution. The current generated by each sensing element can be described as given in:

$$
I=k_{1} \iint \frac{2 E_{l}}{\pi^{2} r} e^{\frac{2\left(x_{1}^{2}+y_{1}^{2}\right)}{r^{2}}} d x_{1} d y_{1}
$$

where $I$ is the current, $r$ the radius of the laser light spot, $E_{t}$ is the energy of the laser beam, $\left(x_{1}, y_{1}\right)$ is the coordinate of a point on the light spot in a coordinates system located at the center of the light spot, and $k_{1}$ is a coefficient. As shown in Fig.4), in the operation region (small neighborhood of the aligned location), the photodiode voltage output $V_{x}$ is approximately linearly related to light intensity units, with a negative slope. As the curve $V_{y}$ is similar to that, it is omitted here. Obviously, when the spot is located in the sensing surface $V_{x} \neq 0, V_{y} \neq 0$ while if the spot is located in the center $\mathrm{V}_{x}=0, V_{y}=0$.
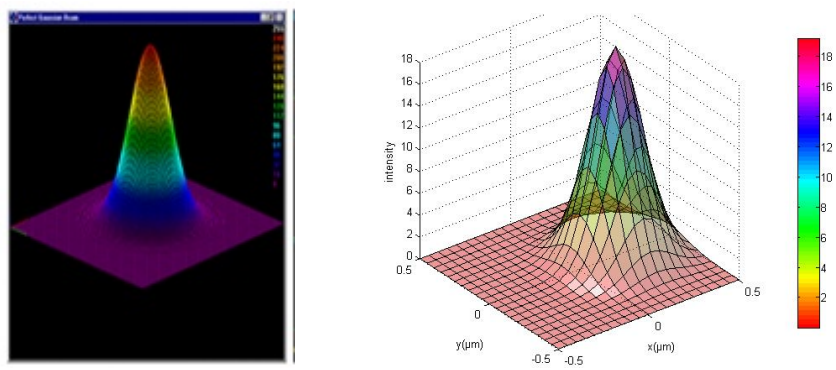

Fig. 5. Light intensity on the laser's beam cross section: (left) theoretical and (right) experimental intensity obeying to Gaussian distribution.

As we can see in Fig.5, the experimental intensity sensed by the PSD can be fitted with a Gaussian distribution as calculated by the theoretical equation (4).

\section{Dynamics of Laser Beam Position}

The laser beam motion is assumed similar to the Brownian motion (represented in Fig. 6) of a particle subjected to excitation and frictional forces. The Brownian motion is given by the generalized differential equation:

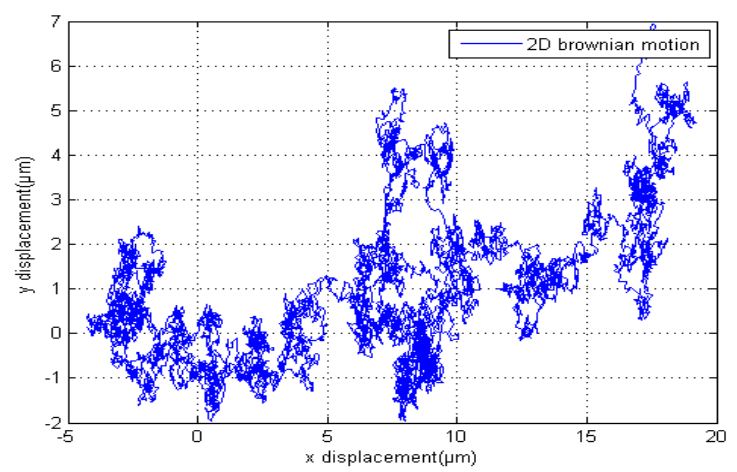

Fig. 6. Particle Brownian motion

$$
\frac{d^{2} x(t)}{d t^{2}}+\beta_{x} \frac{d x(t)}{d t}=W_{x}
$$

were $\beta_{x}$ coefficient of friction and $W_{x} \sim N\left(0, \delta_{x}^{2}\right)$. To estimate with a discrete filter the laser beam positions at each sampling time $t_{k}$, a discrete model of the continuous dynamic (4) is necessary. In the $x$-coordinate the discredited equations of motion using a zero-order hold(zoh) are given by:

$$
\begin{aligned}
& \dot{x}_{k}=\frac{x_{k}-x_{k-1}}{\Delta T} \\
& \ddot{x}_{k}=\frac{\dot{x}_{k}-\dot{x}_{k-1}}{\Delta T}
\end{aligned}
$$

from (4) and (6) we obtain :

$$
\dot{x}_{k}=a_{x} \dot{x}_{k-1}+b_{x} W_{x_{k}}
$$

where $\Delta T$ is the discretisation time step and the statistic properties of the excitation $W_{x_{k}}$ force is assumed to be an zero-mean Gaussian random variable with variance $\delta_{x}^{2}$. The $y$-axis can be modeled in the same manner as the $x$-axis, though with different dynamics. For 2D representation, the source state at discrete time $\mathrm{k}$ is defined as

$$
\left[\begin{array}{llll}
x_{k} & y_{k} & \dot{x}_{k} & \dot{y}_{k}
\end{array}\right]^{T}
$$

$\left(x_{k}, y_{k}\right)$ and $\left(\dot{x}_{k}, \dot{y}_{k}\right)$ are the source position in the plane $x$ $y$ and velocity respectively. The discrete state space of the Brownian laser beam is represented by:

$$
\begin{gathered}
X_{k}=A X_{k-1}+B W_{k} \\
Y_{k}=C X_{k-1}
\end{gathered}
$$


with

$$
\begin{gathered}
A=\left[\begin{array}{cccc}
1 & 0 & \Delta T & 0 \\
0 & 1 & 0 & \Delta T \\
0 & 0 & a_{x} & 0 \\
0 & 0 & 0 & a_{y}
\end{array}\right], B=\left[\begin{array}{cccc}
0 & 0 & b_{x} & 0 \\
0 & 0 & 0 & b_{y}
\end{array}\right]^{T} \\
C=\left[\begin{array}{cccc}
1 & 0 & 0 & 0 \\
0 & 1 & 0 & 0
\end{array}\right]
\end{gathered}
$$

The state representation matrices $(A, B)$ are derived from the particle dynamics defined in (5)-(7)

and $W_{k} \sim N(0, Q)$ is an zero-mean Gaussian random variable with matrix variance $Q$.

It comes from(9) that:

$$
X_{k}=\sum_{i=1}^{k} A^{k-i} B W_{i}+A^{k} X_{0}
$$

Because successive random variables $W_{i}$ form apriori discrete zero mean white Gaussian process, $X_{k}$ form(11) is Gaussian if the knowledge on $X_{0}$ is assumed Gaussian or equal to some fixed value. Its a priori variance at each step $\mathrm{k}$ can be calculated :

$$
\sigma^{2}\left(X_{k}\right)=\sum_{i=1}^{k} A^{k-i} B \sigma^{2}\left(W_{i}\right)+A^{k} \sigma^{2}\left(X_{0}\right)
$$

(12) shows that bigger is the variance of $W_{k}$ to set and bigger is the a priori uncertainty variance on the possible values of the modeled unknown position of the beam laser at $t_{k}$. Finally, the measurement $Y_{k}$ of position takes into account the discrete-time white Gaussian noise $V_{k}$ white zero mean and variance $R$ added by the four quadrant photosensitive detector.

$$
Y_{k}=C X_{k-1}+V_{k}
$$

\section{Control Scheme of Beam Pointing And TRACKING}

In this section we presented the control strategy used to drive PSD for laser beam tracking. The problem considered is that of tracking a laser beam into the $x-y$ plane by robust control issues of the dual micro/nano manipulators motions, and the localization of the current laser beam position [8]. It implies to integrate a prediction-estimation model that anticipates the a priori laser beam motion, taking into account both dynamics of the beam laser and manipulators models.

\section{A. Decoupled Control Structure}

One of the main characteristics of a dual-stage controller there are two control output(micro controller and nano controller). However, one of the constraints must be considered is the control of different dynamics. The first stage actuator, or the micro stage, has a large moving range but a low bandwidth while the second stage actuator, or the nano stage, has a high bandwidth but small moving range. The problem considered how coordinate the micro stage and nano stage

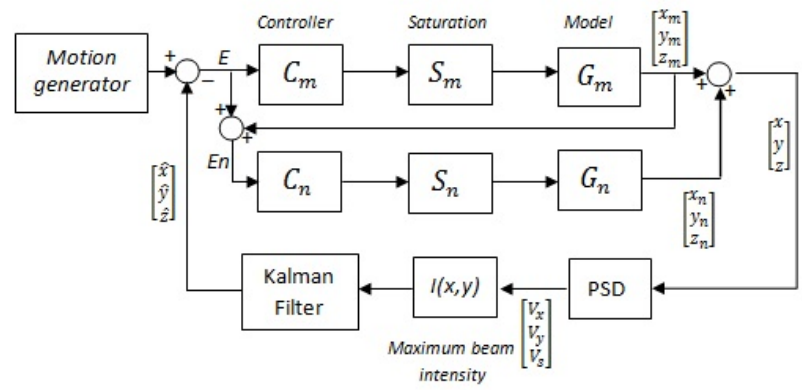

Fig. 7. Master-slave controller with decoupling structure for maximum light tracking.

to tack laser beam by only position feedback signal available delivered by PSD. Which require a control strategy that includes the contribution of both actuators and ensure the performance of each stages. The master-slave control design allow to transform the dual-stage control design problem into decoupled or sequential multiple independent controller designed separately. Fig.7 shows the block diagram of the dual-stage controller using the decoupled control structure. The position error tracking will be compensated by the high bandwidth fine actuator for high precision. The coarse actuator will follow the fine actuator to prevent its saturation.

The total dual-stage open loop transfer function from to, $G_{T}$ is

$$
G_{T}=C_{N} G_{N}+C_{M} G_{M}+C_{N} G_{N} C_{M} G_{M}
$$

and the total closed loop sensitivity function from $R$ to $S$ equals the product of the micro- and nanostage loop sensitivities $, S_{M}, S_{N}$, respectively:

$$
S_{T}=\frac{1}{1+G_{T}}=S_{N} S_{M}
$$

where

$$
S_{N}=\frac{1}{1+C_{N} G_{N}}, S_{M}=\frac{1}{1+C_{M} G_{M}}
$$

That demonstrate the possibility to design the performances of each stage defined by their sensitivity for control synthesis.

\section{B. Kalman Filter Estimator}

In robotics, the Kalman filter is most suited to problems in tracking, localization, and navigation, and less so to problems in mapping [1] [2]. This is because the algorithm works best with well-defined state descriptions (positions, velocities, for example), and for states where observation and time-propagation models are also well understood. The prediction-estimation stages of the Kalman filter are derived from equations (9) and (13):

Prediction. A prediction $\hat{X}_{k \mid k-1}$ of the state at time $\mathrm{k}$ and its covariance $P_{k \mid k-1}$ is computed according to:

$$
\begin{gathered}
\hat{X}_{k \mid k-1}=A \hat{X}_{k-1 \mid k-1}+B U_{k} \\
P_{k \mid k-1}=A P_{k-1 \mid k-1} A^{T}+Q(k)
\end{gathered}
$$


Update. At time $k$ an observation $y(k)$ is made and the updated estimate $\hat{X}_{k \mid k}$ of the state $X_{k}$, together with the updated estimate covariance $P_{k \mid k}$, is computed from the state prediction and observation according to:

$$
\begin{gathered}
\hat{X}_{k \mid k}=\hat{X}_{k \mid k-1}+K_{k}\left(Y_{k}-C_{k} \hat{X}_{k \mid k-1}\right) \\
P_{k \mid k}=P_{k \mid k-1}-K_{k} S_{k} K_{k}^{T}
\end{gathered}
$$

where the gain matrix $K_{k}$ is given by:

$$
K_{k}=P_{k \mid k-1} C_{k} S_{k}^{-1}
$$

where

$$
S_{k}=C_{k} P_{k \mid k-1} C_{k}+R_{k}
$$

is the innovation covariance. The difference between the observation $Y_{k}$ and the prediction observation $C_{k} \hat{X}_{k \mid k-1}$ is termed the innovation or residual $r(k)$. Thus the input of the Kalman filter is the noisy measurement of the laser beam displacement in the $x-Y$ direction delivered by the photodiode detector and $\hat{X}_{k}$ is the output of the filter representing the estimation of the displacement at time $t_{k}$.

\section{EXPERIMENTS}

\section{A. Laser beam position estimation}

A parametrization step is performed in order to get the model parameters of laser beam motion and set parameters of the Kalman filter that used to estimate position of laser beam, based on the experimental results. In this step the PSD is fixed and the laser beam positioning is initiated automatically by moving linearly the laser beam until to be detected by the PSD . After positioning the laser beam in the PSD center, a synthetic trajectory generated randomly (chosen so as does not exceed the workspace of the PSD) is given to the laser beam for local maximum search(see Fig.9). The maximum intensity provided by the PSD is computed by eq(4). The parameters $a_{x}$ and $b x$ are identified by using the equation 8 describing the Brownian motion and the results of experimental intensity variation of the laser beam sensed by the PSD as represented in figure 5 .

The Kalman filter parameters, i.e measurement noise matrix $R$, process noise matrix $Q$ and initial state error matrix $P_{0}$, where chosen as:

$P_{0}=$

$Q=10^{-2} I_{4 \times 4}+\left[\begin{array}{cccc}0 & 2 \mathrm{~b}_{x}^{2} & \mathrm{~b}_{x} & \mathrm{~b}_{x} \\ 2 \mathrm{~b}_{x}^{2} & 0 & \mathrm{~b}_{x}^{2} & \mathrm{~b}_{x}^{2} \\ \mathrm{~b}_{x} & \mathrm{~b}_{x} & 0 & 2 \mathrm{~b}^{3} \\ \mathrm{~b}_{x} & \mathrm{~b}_{x}^{2} & 2 \mathrm{~b}_{x}^{3} & 0\end{array}\right]$

$R=10^{-4} I_{2 \times 2}$

The noise matrices where chosen symmetric and empirically depentended on the coefficient friction $b_{x}$ identified, in order to achieve the best performance of the filter. The results of laser beam motion prediction using Kalman filter are presented in fig.8. At first glance, the filter succeed to follow the true trajectories very closely. As illustrated the performances of the Kalman filter in terms of precision the filter converge to the real position of the laser beam with a minimal error.
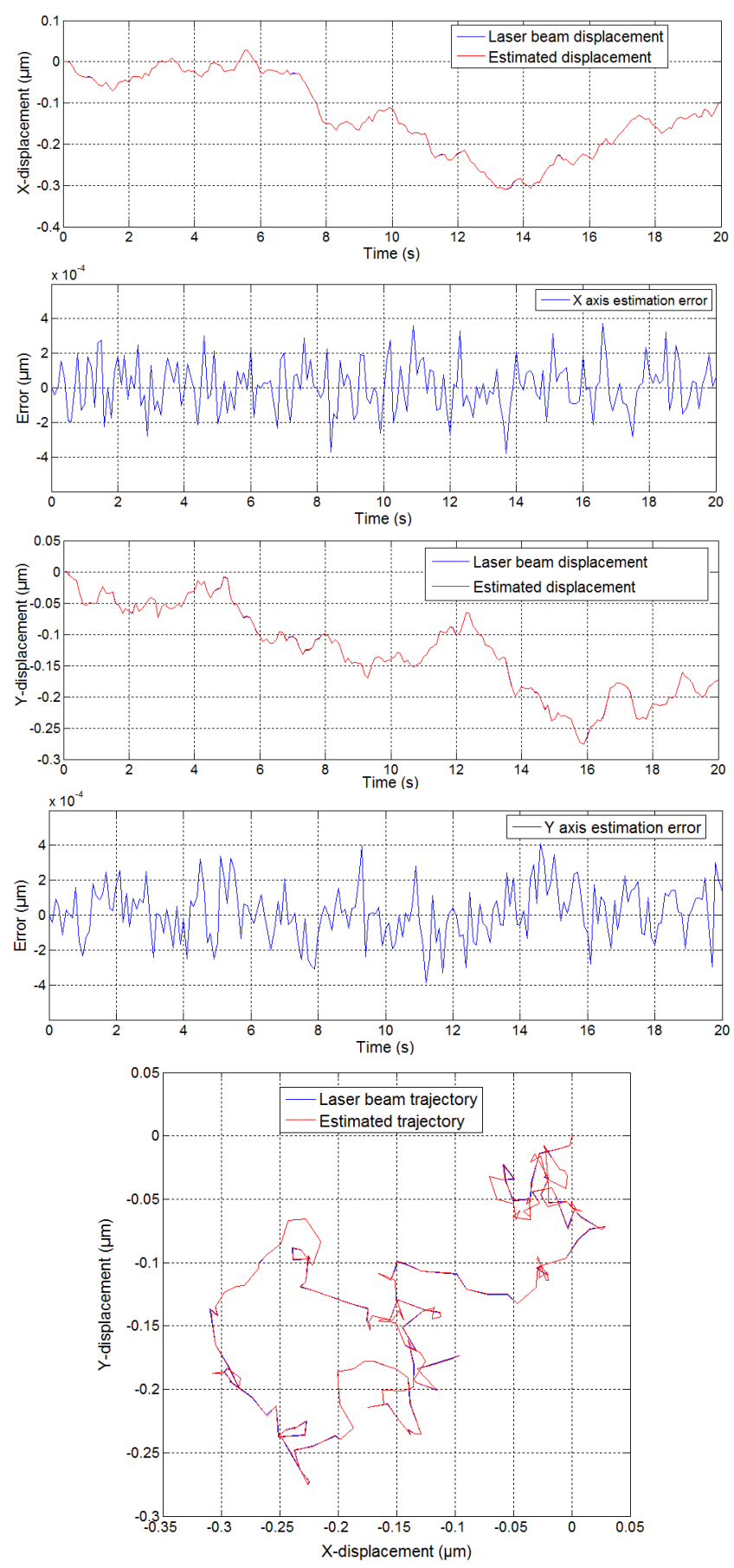

Fig. 8. Experimental tracking with Kalman filter: Motion estimation along $x-y$ axis with corresponding errors and $2 \mathrm{D}$ displacement.

\section{B. Laser beam tracking by piloted PSD}

In a second step, to evaluate the efficient of the Kalman filter used for laser beam position estimation and the control strategy adopted for the second micro-nanostage, to tracks laser beam by the PSD, we give motion to the laser beam, mounted in the fist micro/nanostage by a composite signal (constant displacement and Brownian motion).The results of a typical tracking run for a fast composite signal, realized by the PSD mounted in the second stage is shown in Figures 


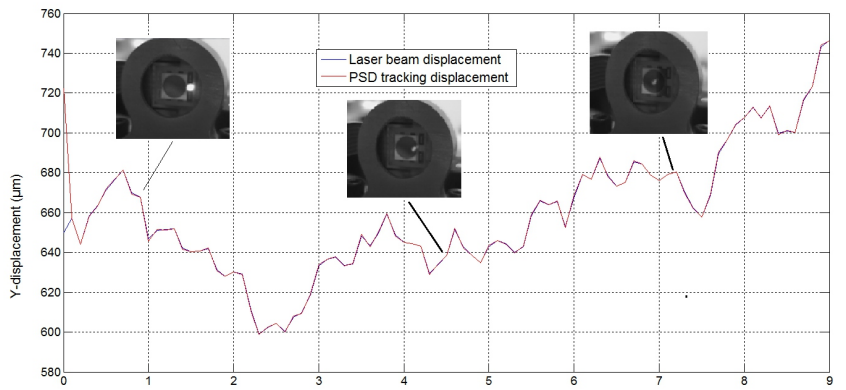

Fig. 9. Laser beam detection of maximum intensity.

11. The resulting motion of the PSD is represented in red color, the blue color represent the composite signal sent to the first micro/nano manipulator.The results demonstrate the robust estimation of the laser beam position is a real time way. As expected, the filtered estimates exhibited a smaller variation. Furthermore, the master-slave controller with decoupled sensitivity is optimized in terms of tracking error as illustrated in the figures the resulting motion of PSD track the composite signal with high precision. It should be noted that this approach assumes variations of laser light intensity during motion, measurement noise, high motion dynamics.

\section{CONCLUSIONS AND FUTURE WORKS}

This paper has presented a study of the control problem of a laser beam illuminating and focusing subjected to dynamic disturbances using light intensity for feedback only. The main idea is to guide and track the beam with a hybrid micro/nanomanipulator which is driven by a control signal generated by processing the beam intensity sensed by a four-quadrant photodiode. The simulations and experiments demonstrated the efficiency of the approach when submitted to external disturbances. The use of the Kalman filter (KF) algorithm for estimating the state of the linear system necessary for implementing the proposed track-following control approach as been proven to be efficient at high dynamics. Further work will be carried out on nanomanipulation of objects under the field of view of a focus laser beam.

\section{REFERENCES}

[1] A. Ehrlicher, T. Betz, B. Stuhrmann, D. Koch, V. Milner, M. G. Raizen, and J. Ks, Proc. Natl. Acad. Sci. U.S.A. 99, 16024 (2002)

[2] B. Stuhrmann, M. Ggler, T. Betz, A. Ehrlicher, D. Koch, and J. Ks, Automated tracking and laser micromanipulation of motile cells, Rev. Sci. Instrum. 76, 035105 (2005)

[3] H. Xie , S. Rgnier, High-Efficiency Automated Nanomanipulation with Parallel Imaging/Manipulation Force Microscopy, IEEE Transactions on Nanotechnology, Vol.11, Iss.1, pp:21-33, 2012.

[4] Pawel K. Orzechowski, James S. Gibson, Tsu-Chin Tsao, Optimal Disturbance Rejection by LTI Feedback Control in a Laser Beam Steering System, 43rd IEEE Conference on Decision and Control, December 14-17, 2004, Atlantis, Paradise Island, Bahamas, pp. 21432148.

[5] C.C. Lee, J. Park, Temperature Measurement of Visible Light-Emitting Diodes Using Nematic Liquid Crystal Thermography With Laser Illumination, IEEE Photonics Technology Letters, Vol. 16, NO. 7, July 2004.
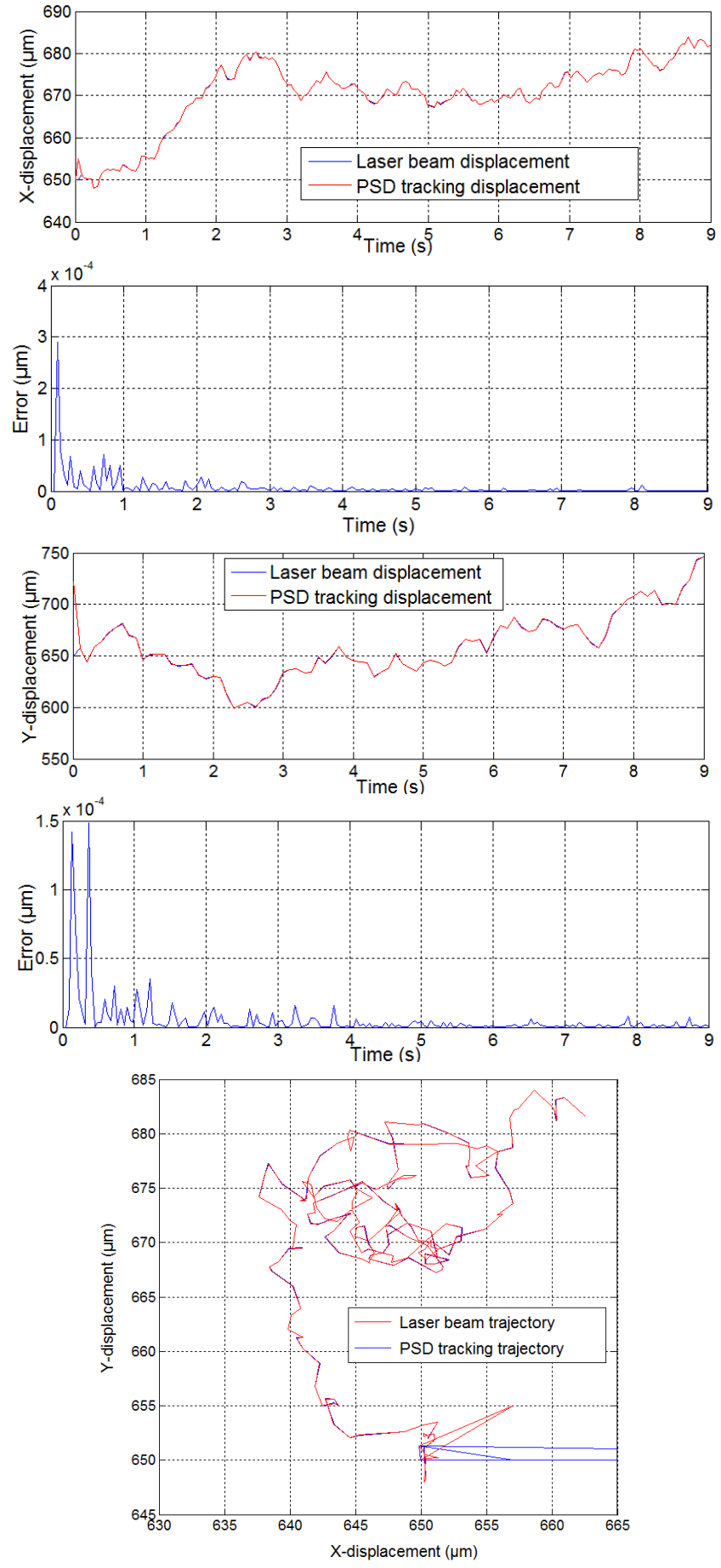

Fig. 10. Experimental tracking with Kalman filter: Motion estimation along $x-y$ axis with corresponding errors and 2D displacement.

[6] R. B. Evans, J.S. Griesbach, W.C. Messner, Piezoelectric microactuator for dual-stage control, IEEE Transactions on Magnetics, Vol.35, pp.977-981,1999.

[7] Nstor O. Prez-Arancibia, James S. Gibson, and Tsu-Chin Tsao, Observer-Based Intensity-Feedback Control for Laser Beam Pointing and Tracking, IEEE Transactions on Control Systems Technology, Vol.20, No.1, 2012, pp.31-47.

[8] Tran Trung Nguyen, A. Amthor and C. Ament, High Precision Laser Tracker System for Contactless Position Measurement, 2011 IEEE International Conference on Control System, Computing. 